\title{
"Nanohybrids" Based on pH-Responsive Hydrogels and Inorganic Nanoparticles for Drug Delivery and Sensor Applications
}

\author{
Andreas Riedinger, ${ }^{\dagger}$ Manuel Pernia Leal, ${ }^{\dagger}$ Smriti R. Deka, ${ }^{\dagger}$ Chandramohan George, ${ }^{\dagger}$ Isabella R. Franchini, ${ }^{+,}$ \\ Andrea Falqui, ${ }^{+}$Roberto Cingolani, ${ }^{\dagger}$ and Teresa Pellegrino ${ }^{*,+,}$ \\ ${ }^{\dagger}$ Istituto Italiano di Tecnologia, Via Morego 30, 16163 Genova, Italy \\ ${ }^{\ddagger}$ Nanoscience Institute of CNR, National Nanotechnology Laboratory, Via Arnesano, 73100 Lecce, Italy \\ Supporting Information
}

ABSTRACT: Allyl-PEG capped inorganic NPs, including magnetic iron oxide (IONPs), fluorescent $\mathrm{CdSe} / \mathrm{ZnS}$ quantum dots (QDs), and metallic gold (AuNPs of 5 and $10 \mathrm{~nm}$ ) both individually and in combination, were covalently attached to $\mathrm{pH}$ responsive poly(2-vinylpyridine-co-divinylbenzene) nanogels via a facile and robust one-step surfactant-free emulsion polymerization procedure. Control of the NPs associated to the nanogels was

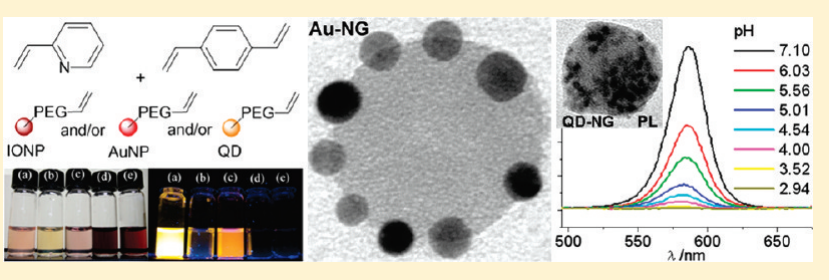
achieved by the late injection of the NPs to the polymerization solution at a stage when just polymeric radicals were present. Remarkably, by varying the total amount of NPs injected, the swelling behavior could be affected. Furthermore, the magnetic response as well as the optical features of the nanogels containing either IONPs or QDs could be modified. In addition, a radical quenching in case of gold nanoparticles was observed, thus affecting the final nanogel geometry.

KEYWORDS: pH responsive hydrogels, quantum dots, superparamagnetic nanoparticles, gold nanoparticles, surfactant freeemulsion polymerization, vinylpyridine, divinylbenzene

\begin{abstract}
Ctimuli-responsive polymer nanogels (NGs) are promising $\checkmark$ materials for drug delivery applications because of their trigger-assisted properties; the $\mathrm{pH}$ or temperature dependent swelling behavior of "smart" polymers enables the controlled loading and release of drugs or other payload molecules associated with the polymer. ${ }^{1-6}$ Their combination with inorganic NPs results in nanostructures with additional functional properties. For example, magnetic manipulation can be achieved if superparamagnetic iron oxide NPs (IONPs) are incorporated, bright photoluminescence is obtained if QDs are bound to the NGs and plasmonic properties are gained when linking metallic NPs, such as gold NPs. The most common techniques used to synthesize nanoscaled polymeric NGs are emulsion (and inversed emulsion), mini-emulsion (and inversed mini-emulsion), or precipitation polymerizations. ${ }^{7-9}$ By these methods high yields of polymer NGs with narrow size distributions with sizes practically tunable at the mesoscale (below $200 \mathrm{~nm}$ ) can be achieved. To integrate inorganic NPs into polymer NGs different strategies can be exploited, such as the physical entrapment, coprecipitation methods, in situ synthesis, and the covalent linkage. ${ }^{10-14}$ Among them, in order to preserve the properties of both the inorganic NPs and the NGs and to have stable and robust functionalized NGs, covalent linkage is perhaps the most appropriated procedure. Also, by this method various types of inorganic NPs might be incorporated simultaneously within the same NGs as here shown.
\end{abstract}

Here, we report a synthetic approach to prepare $\mathrm{pH}$-responsive poly(2-vinylpyridine-co-divinylbenzene-) NGs covalently functionalized with inorganic NPs. The late injection of allyl-functionalized inorganic NPs during emulsion polymerization allows us to control the incorporation into the NGs of different types of inorganic NPs while preserving the acidic $\mathrm{pH}$ response of the NGs. Copolymerization of the 2-vinylpyridine (2VP) and divinylbenzene (DVB) by surfactant-free emulsion polymerization has been previously described ${ }^{8}$ and the size tunability of the 2VP-DVB NGs in the range of $40-200 \mathrm{~nm}$ has been demonstrated. ${ }^{11}$

In order to copolymerize the inorganic NPs with the NGs, the NPs must have accessible $\mathrm{C}-\mathrm{C}$ double bonds at their surface on which the radical reaction can be propagated. Also, the NPs should be water-soluble since water is the reaction medium in which the NPs are injected, and they should also be stable in the oil phase in which the formation and the growth of the polymeric NGs occur. In order to meet these criteria, the transfer of the inorganic NPs to aqueous solution was achieved by the functionalization with poly(ethylene glycol) (PEG) molecules as the NPs were originally synthesized via colloidal methods involving nonpolar solvents. ${ }^{15-17}$ We chose PEG molecules bearing an allyl group as polymerizable entity at one end and a binding unit at the other end which is reactive toward the different NP surfaces (HS-PEG-allyl and Gallol-PEG-allyl, see Figure 1A, B). At the same time, the PEG chain guarantees solubility of the NPs both in the oil and in the aqueous phase, due to its amphiphilic character. While the PEG-allyl portion of the ligand could be the

Received: April 7, 2011

Revised: June 15, 2011

Published: June 21, 2011 


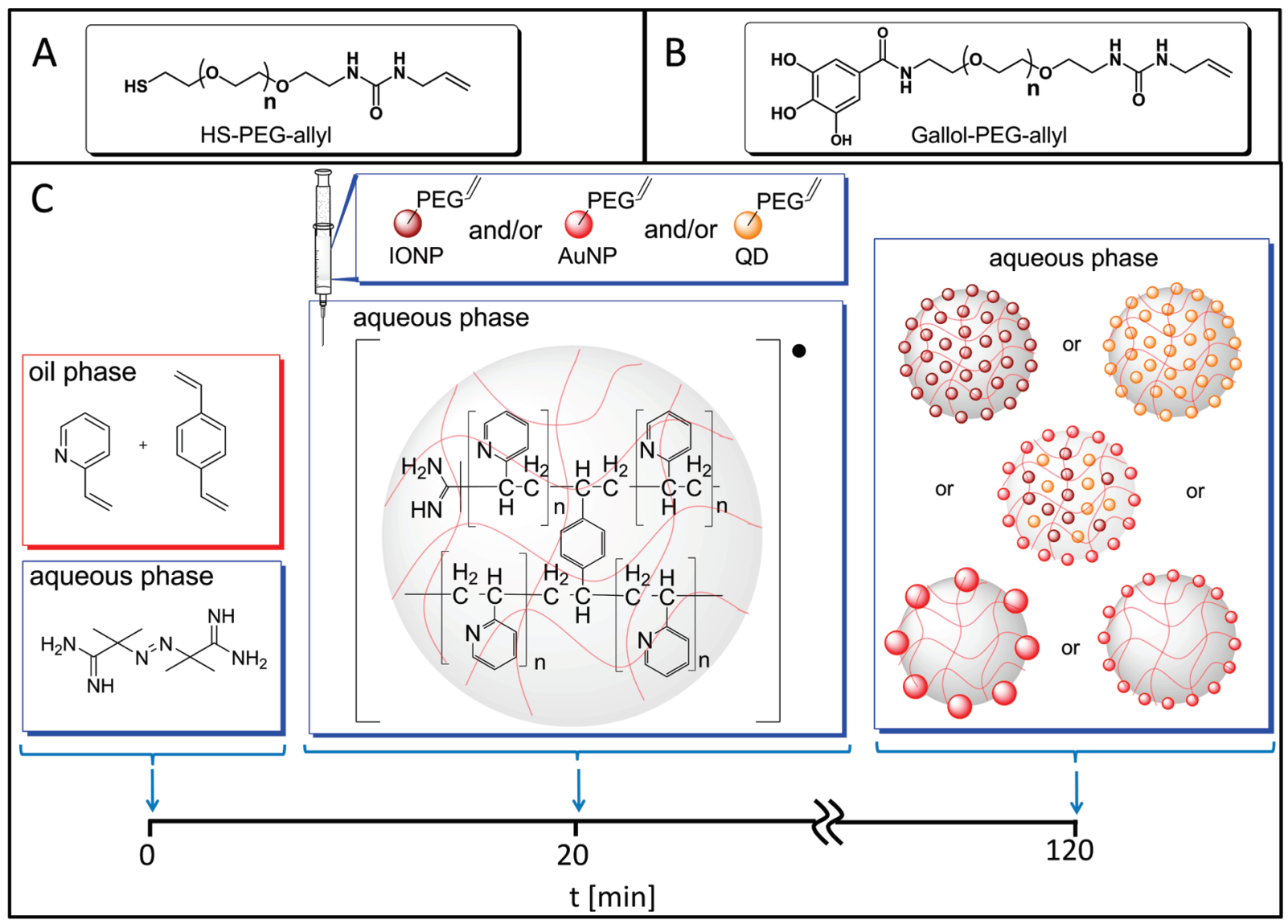

Figure 1. Time line scheme for the copolymerization of 2VP/DVB with IONPs/QDs/AuNPs@X-PEG-Al by late injection emulsion polymerization. The monomers 2VP and DVB (oil phase) are emulsified in continuous aqueous phase containing the initiator (AIBA). After 20 min the radical containing species in the aqueous phase are mostly polymeric radicals. By fast injection of the PEG-allyl-functionalized NPs, polymer/nanoparticle composite nanogels can be obtained with tunable solid content and different NPs distribution of the polymeric beads.

same for any of the core material used, the NP binding moiety was properly selected depending on the type of NP. For Au NPs and QDs, mercapto-modified PEG allyl molecules (HS-PEG-Al, Figure 1A, Supporting Information, for ligand synthesis and NPfunctionalized characterization) were exchanged at the NP surface, as the thiols can strongly bind to both gold atoms as well as to the $\mathrm{ZnS}$ shell of $\mathrm{CdSe} / \mathrm{ZnS}$ core-shell QDs. ${ }^{18}$ Instead, for IONPs, gallol-PEG allyl molecules (Figure 1B and Supporting Information) were chosen as it is known that the catechol derivates can complex well with the accessible $\mathrm{Fe}^{2+} / \mathrm{Fe}^{3+}$ ions at the IONP surface. ${ }^{19,20}$

The preparation of $2 \mathrm{VP} / \mathrm{DVB}$ NGs functionalized with NPs of different materials reported here exploits a simple concept: in a one-step reaction, the addition of the allyl-PEG functionalized NPs to the reaction mixture occurs at least $20 \mathrm{~min}$ after the polymerization has started. This time delay is required in order to allow first the nucleation of the polymer NGs and at the same time the consumption of the free initiators and most of the monomers in the water phase, thus maximizing at 20 min the probability for the NPs to react toward the polymeric radicals at the surface of the growing NGs (see Figure 1C). Indeed, it is critical to prevent that the allyl-NPs, which are also soluble in water, act as nuclei themselves on which the polymerization can initiate and proceed independently from that of the NGs. Therefore, the delay time (20 min was found the most suitable time within the different time lapses tested) was indeed necessary to start the polymerization, leading first to the nucleation of 2VB/DVB beads having hydrodynamic diameters of about $25 \mathrm{~nm}$ (checked by DLS, see Supporting Information, Figure S10), and to the following copolymerization of the allyl-capped NPs. Under these conditions, at the end of the reaction time (120 $\mathrm{min}$ ) a homogeneous reaction product composed of NGs functionalized with NPs was formed (100\% of the NPs could be successfully grafted onto the polymer NGs and no free NGs or free NPs were found in the final product, see Figure 2 and Figure S7, SI). On the contrary, if the NPs, the 2VP and DVB, were mixed all together at the start of the reaction and then the polymerization reaction was carried out, a more inhomogeneous sample was obtained in which many empty NGs were also present in addition to the targeted NGs functionalized with NPs.

While with IONPs and QDs the amounts of NPs inserted into the NGs could be varied in a wide range (between ca. 6 and $800 \mathrm{nM}$ for either IONPs or QDs as determined by ICP, see Supporting Information), in the case of Au NPs the amount incorporated was limited to a narrow range (around $5 \mathrm{nM}$ for

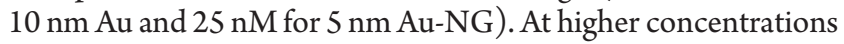
of Au NPs than these critical limits, the NPs were not copolymerized and instead were found free in solution. Also, the geometry of the resulting NGs was related to the type of NPs that were incorporated (Figure 2). While the IONPs and the QDs NPs were covering more homogenously the NGs, the Au NPs displayed a corona-like covering as seen in dried state on the 


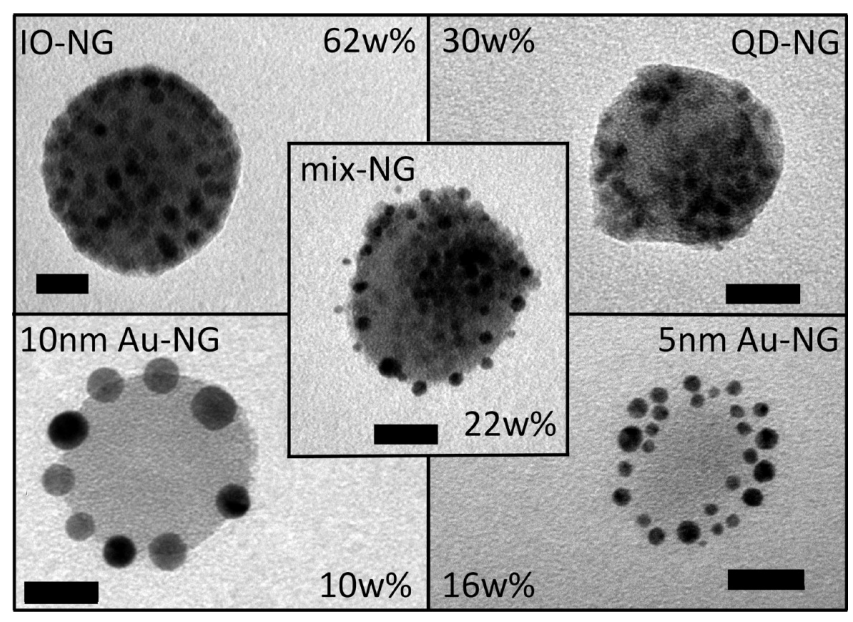

Figure 2. Characteristic TEM micrographs at $\mathrm{pH} 7$ of $2 \mathrm{VP} / \mathrm{DVB}$ NGs including IONPs (IO-NG), QDs (QD-NG), $10 \mathrm{~nm}$ AuNPs (10 nm Au$\mathrm{NG}$ ), $5 \mathrm{~nm}$ AuNPs ( $5 \mathrm{~nm} \mathrm{Au-NG)} \mathrm{and} \mathrm{NGs} \mathrm{containing} \mathrm{IONPs/QDs/}$ $5 \mathrm{~nm}$ AuNPs (mix-NG). The scale bars correspond to $20 \mathrm{~nm}$. The weight percentage numbers indicate the inorganic solid content measured by thermal gravimetric analysis, TGA (see also Supporting Information).

transmission electron microscopy (TEM) grid, in which the Au NPs were distributed mostly on the edge of the NGs. In order to confirm the role of $\mathrm{Au}$ (at high concentrations) that hinders the formation of NGs, the synthesis of NGs was carried out in the presence of $\mathrm{Au}$ NPs capped with PEG molecules bearing a methoxy-termination, thus without the allyl group (HS-PEG$\mathrm{OMe}$ ). In this case, no copolymerization of Au NPs with the NGs was expected and if no Au interference occurred, empty NGs together with free single Au NPs should be obtained in the final sample. Nevertheless, TEM analysis showed that no NGs were formed in the presence of methoxy-PEG-capped Au NPs in the reaction mixture (at $\mathrm{Au}$ concentration exceeded $60 \mathrm{nM}$ ), thus confirming the inhibition of NG formation by the metallic core (Au). Most likely, the Au NPs can easily sequester electrons from the radical chains of the polymer resulting in a quenching of radical product (and leading for example to an elimination product). This effect was dependent on the Au concentration, since upon increasing the Au NPs amount the polymerization was quenched and no NGs were formed. It is worth to note however that for the two sizes of Au NPs chosen, the total Au NP surface area (calculated by considering the concentrations at which the $\mathrm{Au}$ NGs could be still obtained, see Supporting Information) has similar values for both sizes of Au NPs, thus indicating that the total accessible area of Au NPs exposed (more than the concentration of nanoparticles itself) is the critical parameter, promoting the incorporation of AuNPs within the NGs. The quenching effect of Au NPs was also confirmed by measuring the monomer conversion percentage. After $20 \mathrm{~min}$ of the reaction time the monomer conversion was found to be around $60 \%$ before injecting the NPs. After $120 \mathrm{~min}$, the conversion percentages were found basically unchanged for all the NGs containing $\mathrm{Au}$ ( $5 \mathrm{~nm}$ Au-, $10 \mathrm{~nm} \mathrm{Au-} \mathrm{and} \mathrm{mix-NGs),}$ while in case of QD-NG and IO-NG the monomer conversion reached values between 90 and 95\% (see Supporting Information). This shows clearly that the addition of a sufficient amount of $\mathrm{Au}$ NPs to the ongoing reaction quenches the polymerization completely. These results are consistent with the findings obtained by Sheibat-Othman et al. when silica particles were used in the surfactant free emulsion polymerization of poly(styrene-co-methyl methacrylate); even in this case if critical threshold concentrations of silica particles were used also the quenching of the polymerization was observed. ${ }^{21}$ The critical $\mathrm{Au}$ concentration together with the partially conversion of the monomers in the Au NGs might also explain the corona-like geometry of $\mathrm{Au}-\mathrm{NGs}$; the $\mathrm{Au}-\mathrm{NGs}$ with less rigid nanostructures can arrange in a corona-like nanostructures during the drying process on the TEM grid. Duguet et al. have observed similar results in silica-polystyrene multipod composits. ${ }^{22}$ When the monomer conversion was far from $100 \%$ due to a less rigid nanostructure obtained, the arrangement of the polystyrene around the silica beads lead to a corona-like structures observed under TEM (the authors also showed that for samples after full conversion did not show this effect; the system was rigid enough to remain once deposited on the substrate in the same geometry as found in solution). ${ }^{23}$

The $\mathrm{pH}$ response of the samples shown in Figure 3 was assessed by dynamic light scattering (DLS, Figure 3A) and was confirmed by TEM analysis (Figure 3B, Supporting Information). As for the NGs alone, the volume transition occurred always at a $\mathrm{pH}$ around 4 and depending on the amount of NPs included into the NGs (as determined by thermal gravimetric analysis (TGA) and inductively coupled plasma (ICP), see Supporting Information), a general trend could be observed: the higher the concentration of NPs copolymerized with 2VP/ $\mathrm{DVB}$, the lesser was the change in the volume transition. There are at least three possible reasons for this behavior. First, the copolymerized NPs generate steric hindrance that results in a more rigid NG surface with a corresponding loss in volume transition. Additionally, if more NPs were copolymerized with the NGs a more cross-linked NG structure might result (each NP surface has multiple $\mathrm{C}-\mathrm{C}$ double bonds and each of them can be copolymerized with multiple polymer chains of the NG). Finally, the NPs can also copolymerize with each other, thus contributing to less permeable NGs.

Investigations of the photoluminescence (PL) of the QD-NG at various $\mathrm{pHs}$ showed an interesting effect. The bright fluorescence of the QD-NG exhibited at $\mathrm{pH} 7$ was quenched when the $\mathrm{pH}$ was lowered and it was lost completely and irreversibly at $\mathrm{pH} 3$ (Figure 4). The $\mathrm{ZnS}$ shell, which is usually composed of a few atomic layers, is likely degraded in acidic conditions (below $\mathrm{pH} 5$ ), resulting in a reduced passivation of the CdSe and consequently to the fast quenching of the fluorescence as already reported in the literature. ${ }^{24-26}$ This indeed is observed even on our system when measuring by ICP the Cd ion release in solution at different $\mathrm{pH}$ after separation of the QD-NGs from water by centrifugation in a filter (molecular cutoff point $=100 \mathrm{kDa}$, see Figure 4B).

The remarkable quenching effect in our system can be already seen at $\mathrm{pH}$ 6. At this $\mathrm{pH}$, degradation of the $\mathrm{ZnS}$ is quite unlikely, which is confirmed by the fact that the PL signal stays constant over days and no significant dissolution of Cd was found for QDNGs at pHs between 5 and 7. The observed loss in PL intensity at pHs between 7 and 5 must be therefore related to another phenomenon. Mulvaney et al. demonstrated that by applying a positive potential, thus injecting positive charges (holes, $\mathrm{h}^{+}$) on $\mathrm{CdSe} / \mathrm{ZnS}$ QDs deposited on a Au substrate, the PL intensity of the QDs was irreversibly quenched. The authors suggested that likely if a positive potential is applied the injected holes might induce a chemical oxidation at the $\mathrm{CdSe} / \mathrm{ZnS}$ interface $(\mathrm{CdSe}+2$ $\left.\mathrm{h}^{+} \rightarrow \mathrm{Cd}^{2+}+\mathrm{Se}\right)$. The products of the reaction, the $\mathrm{Cd}^{2+}$ ions and 

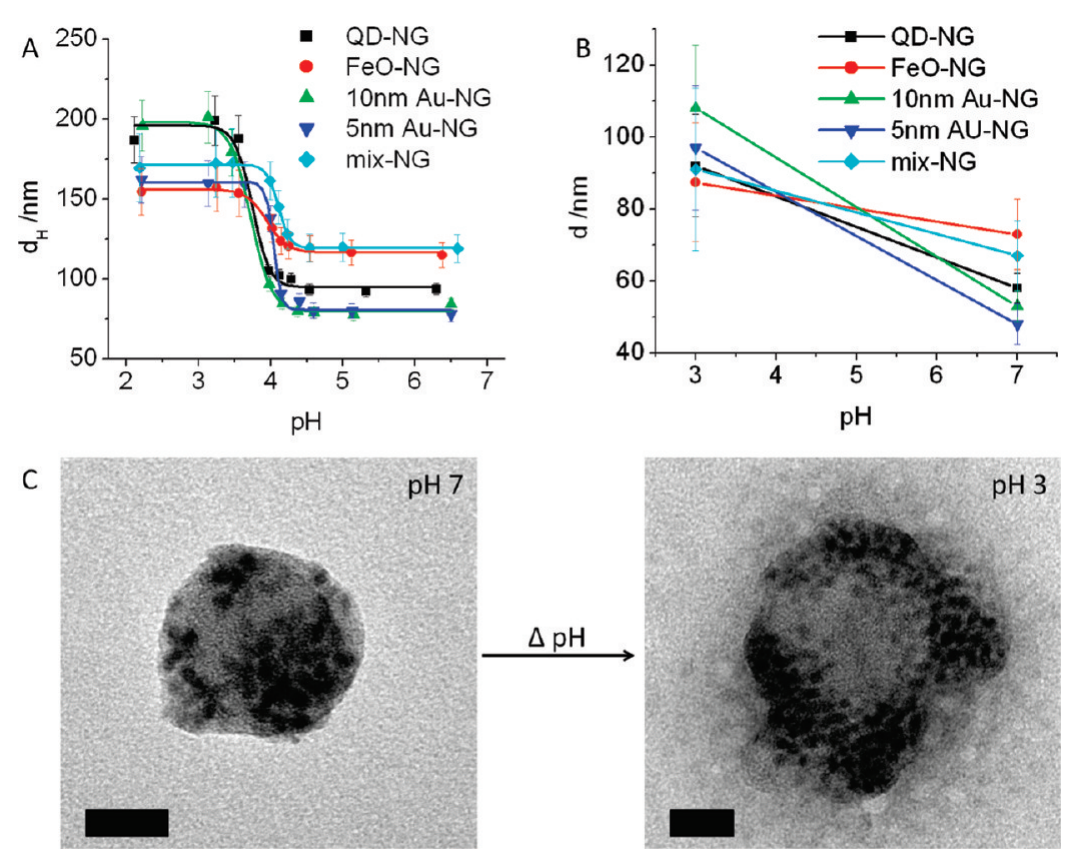

Figure 3. $\mathrm{pH}$ response of the NP-NGs. (A) Hydrodynamic diameters $\mathrm{d}_{\mathrm{H}}$ (intensity mean measured by DLS) of the different NP-functionalized NGs at different $\mathrm{pHs}$ (see also for additional DLS data). Error bars reflect the SD. (B) TEM diameters of the NP-functionalized-NGs at pH 7 and pH 3 (the average values were calculated on an average of $100 \mathrm{NGs}$ measured; the NGs at $\mathrm{pH} 3$ were stained with $1 \%$ aqueous OsO4 solution). Error bars reflect the SD. (C) Typical TEM micrograph of NP-NG (in the specific case QD-NG) at $\mathrm{pH} 7$ and $\mathrm{pH} 3$ (right image: $\mathrm{NG}$ was stained with $1 \%$ aqueous OsO solution). The scale bars correspond to $20 \mathrm{~nm}$. NPs are covalently attached to the NG and thus are not released at pH 3.The TEM images of NGs shown are representative of the samples but do not correspond to each other.
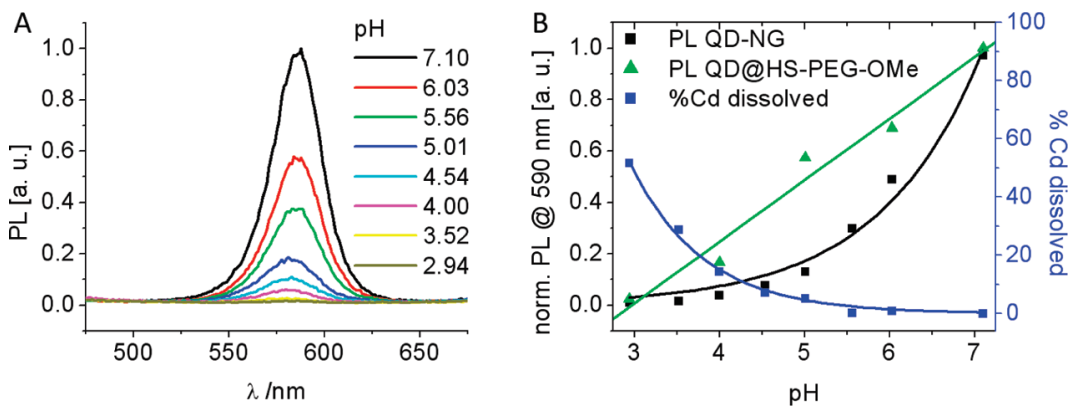

Figure 4. (A) Photoluminescence of QD-NGs at various $\mathrm{pHs}$. The bright QD-NG pholuminescence at $\mathrm{pH} 7$ is quenched when the $\mathrm{pH}$ is lowered to pH 3. (B) Plot of the PL at $590 \mathrm{~nm}$ versus the pH for the QD-NG and the QDs@HS-PEG-OMe and the Cd found in downstream of the QD-NG at different $\mathrm{pHs}$ by after filtering in centrifuge filter. The difference in the slope of the PL decay indicates a lower $\mathrm{pH}$ at the NGs surface. Quenching of fluorescence of QD-NG at $\mathrm{pH}$ lower than 7 allows $\mathrm{pH}$ sensing from $\mathrm{pH} 7$ to $\mathrm{pH} 4$ at which the swelling of the NGs starts. Going from $\mathrm{pH} 7$ to $\mathrm{pH} 5.5$ ca. $70 \%$ of fluorescence is quenched while almost no free $\mathrm{Cd}$ could be detected by ICP. For $\mathrm{pH}$ lower than 5 a significant Cd release can be detected.

Se atoms lattice, confined at the $\mathrm{CdSe} / \mathrm{ZnS}$ interface, act as trap states for the electrons and the holes and thus could play the role of dark recombination centers. ${ }^{27}$ In analogy to the Mulvaney findings, we are likely observing a similar behavior except that the positive charges are injected by the protonation of the pyridine of the nanogel network associated to the QDs. We assume that in case of QD-NGs, the steep exponential PL decay with decreasing the $\mathrm{pH}$ is related to the local environment at which the QDs, included into the NGs, are exposed. At pH 7, the NG network is well compacted around the QDs. This screens the QD surface from the external environment and results in NGs with a bright fluorescence. When the $\mathrm{pH}$ is lowered, the pyridine units of the polymer chains attract protons and the local proton concentration (positive charge density) in the proximity of the QDs linked to the NGs is higher than in the bulk solution thus resulting in a locally lower $\mathrm{pH}$ and consequent $\mathrm{PL}$ decay. ${ }^{28}$ For the QDs capped with HS-PEG-allyl alone the same effect was observed, but the decrease of PL was linear with the $\mathrm{pH}$ and thus the effect of PL intensity loss was less dramatic. Here, it is remarkable the difference in the trend of degradation of the same allyl-PEG-QD when they were covalently linked to NGs or when they were free in solution. The association with a $\mathrm{pH}$ responsive NGs can significantly affects their local $\mathrm{pH}$ environment and thus the PL decay.

These findings make both the mix-NGs and the QD-NGs interesting for potential optical $\mathrm{pH}$ sensors in the range from 7 to 4 (see Figure 4B). To cite one example, for tracking the cellular delivery of the cargo associated to the $\mathrm{pH}-\mathrm{NG}$ (which should 


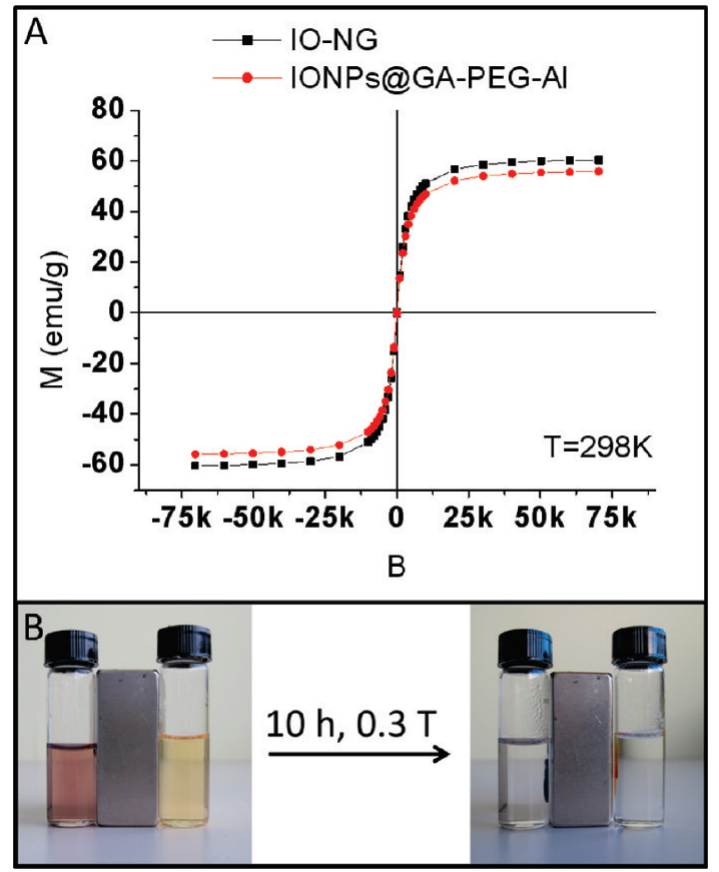

Figure 5. (A) $\mathrm{M}(\mathrm{H})$ curves of IO-NPs and IO-NGs at 298K. (B) NGs with incorporated IO NPs can be quantitatively collected by a small magnet $(0.3 \mathrm{~T})$ after $10 \mathrm{~h}$. Left vial, mix-NG; right vial, IO-NG.

occur when the nanogel is in a swelling state and thus below $\mathrm{pH}$ 5) the QD-NGs can be applied as in situ $\mathrm{pH}$ sensors to detect at subcellular level the pathway and the localization of the NGs within the different cellular compartments. Indeed it is known that the early endosomes have a $\mathrm{pH}$ around $6-5.5$ and at these pHs the QD-NGs preserve partially the fluorescence, while the $\mathrm{pH}$ of late endosomes and lysosomes goes below $4.5-4$ and at these $\mathrm{pH}$ values the fluorescent of the QD-NGs is lost almost completely. ${ }^{29,30}$

Optical properties of $5 \mathrm{~nm}$ Au-NG and $10 \mathrm{~nm} \mathrm{Au-NG}$ at $\mathrm{pH} 7$ and $\mathrm{pH} 3$ were also investigated by $\mathrm{UV}$-vis spectroscopy (see Supporting Information). If the Au-NGs were brought to $\mathrm{pH} 3$, the polymer swelled and the average distance between the AuNPs was increased. Indeed, a small blue shift of $3 \mathrm{~nm}$ (toward shorter wavelengths) of the typical Au plasmon peak at $520 \mathrm{~nm}$ was observed at $\mathrm{pH} 3$ with respect to $\mathrm{pH} 7$. Also, it is worth mentioning that the absorption spectrum of mix-NG at $\mathrm{pH} 7$ shows a broad absorption peak derived from the merging of the plasmon peak of the Au-NPs and the absorption of the QDs (see Supporting Information). For the mix-NG, at $\mathrm{pH} 3$, while the plasmon peak of the Au-NGs was slightly blue shifted, the absorption of the QD-NGs was unchanged while the PL peak at $590 \mathrm{~nm}$ was quenched (see Supporting Information, Figure S13).

Finally, all nanogels including magnetic nanoparticles (mixNG, IO-NG) could be quantitatively collected using an external magnet $(0.3 \mathrm{~T})$ within few hours (at a concentration of IONPs, $6 \mathrm{~nm}$ in diameter, between 140 to $800 \mathrm{nM}$, see Figure 5B), thus indicating that the magnetic moments of the single IONPs are summed up in the NGs allowing a faster magnetic separation compared to that of single IONP while preserving the superparamagnetic behavior of the IONPs. The $\mathrm{M}(\mathrm{H})$ curves recorded show clearly that the superparamagnetic behavior of the IONPs is kept even when the IONPs are incorporated into the NG since no hysteresis loop is observed for the IO-NGs at $298 \mathrm{~K}$
(Figure 5A). The force exerted on an IO-NG can be calculated with the well-known eq $1^{31}$

$$
F_{\mathrm{m}}=\nabla(\mu H)
$$

where $H$ is the magnetic field and $\mu$ is the single IONPs magnetic moment. In the case of IONPs, this force is then exerted on each single particle (moment: $\mu$ ) and the corresponding movement toward the magnet has to compete, as first approximation, with the Brownian motion in solution.

In the case of the sample IO-NG, the total magnetic moment $\left(\mu_{\mathrm{T}}\right)$ that has to be taken into account to apply the eq 1 is that obtained as vectorial sum of all the single magnetic nanoparticle moments over the total volume of the single nanogel (see Supporting Information). By an approximate calculation we found that in the case of the IO-NGs this force scales with a factor of 27 times higher than that of the single IONPs, thus allowing a faster quantitative accumulation of the IO-NGs to the $0.3 \mathrm{~T}$ magnet as experimentally observed (Figure 5B).

In summary, we described a facile synthetic approach to prepare $\mathrm{pH}$ responsive poly(2-vinylpyridine-co-divinylbenzeneco-nanoparticles) composite NGs via late injection emulsion polymerization. Various nanoparticles (IONPs, QDs, and AuNPs) could be covalently attached to the NGs. To the best of our knowledge, the arbitrary combination of different inorganic NPs with $\mathrm{pH}$ responsive monomers via one pot emulsion copolymerization, as well as the late injection to solution/suspension of radical polymeric nanogels has not yet been reported. With this approach, the different types of the inorganic NPs and the different comonomers that compose the nanogels could be arbitrarily chosen, thus extending the procedure to the fabrication of different polymer/inorganic NPs nanocomposites. While the $\mathrm{pH}$-responsive behavior of the NGs is kept even when the inorganic NPs are incorporated within the NGs thus suggesting possible exploitation of the system as carrier for different charged payloads, the close proximity of the nanoparticles to the NG network influences the NP properties. In the case of the gold NPs, the radical reaction and the geometry of the Au-NGs obtained were affected, while in the case of the QDs the dependence of the photoluminescence decay of the QD-NGs was drastically changed with respect to free QDs and this feature can be exploited to sense the local $\mathrm{pH}$ at the QD surface. Finally, the multifunctional nanogels here developed might find applications in biomedicine as multitasks tools for parallel (i) $\mathrm{pH}$ sensors, ${ }^{32}$ (ii) heating agents (due to the magnetic NPs) for hyperthermia treatment, ${ }^{33,34}$ and (iii) nanocontainers for different drug or payloads (short interfering RNA) with release triggered by the acidic $\mathrm{pH}^{30}$

\section{ASSOCIATED CONTENT}

S Supporting Information. Experimental section, monomers conversion experiments, NMR spectra of ligands, additional TEM images, DLS, TGA analysis, UV-vis and PL spectra, ZFC, FC magnetic measurements, and FT-IR of the NPs and of the NGs are also reported. This material is available free of charge via the Internet at http://pubs.acs.org.

\section{AUTHOR INFORMATION}

\section{Corresponding Author}

*E-mail: teresa.pellegrino@unile.it. 


\section{ACKNOWLEDGMENT}

This work was supported in part by the European project Magnifyco (Contract NMP4-SL-2009-228622). M.P.L. thanks to the "Fundación Progreso y Salud" for the postdoctoral fellowship. We thank Giammarino Pugliese for providing Iron Oxide NPs and Liberato Manna for helpful discussion.

\section{REFERENCES}

(1) Talsma, S. S.; Babensee, J. E.; Murthy, N.; Williams, I. R. J. Controlled Release 2006, 112, 271-279.

(2) Sisson, A. L.; Steinhilber, D.; Rossow, T.; Welker, P.; Licha, K.; Haag, R. Angew. Chem., Int. Ed. 2009, 48, 7540-7545.

(3) Bachelder, E. M.; Beaudette, T. T.; Broaders, K. E.; Dashe, J.; Freİchet, J. M. J. J. Am. Chem. Soc. 2008, 130, 10494-10495.

(4) Kakizawa, Y.; Harada, A.; Kataoka, K. J. Am. Chem. Soc. 1999, 121, 11247-11248.

(5) Oh, J. K.; Drumright, R.; Siegwart, D. J.; Matyjaszewski, K. Prog. Polym. Sci. 2008, 33, 448-477.

(6) Kabanov, A. V.; Vinogradov, S. V. Angew. Chem., Int. Ed. 2009, 48, 5418-5429.

(7) Peppas, N. A.; Hilt, J. Z.; Khademhosseini, A.; Langer, R. Adv. Mater. 2006, 18, 1345-1360.

(8) Loxley, A.; Vincent, B. Colloid Polym. Sci. 1997, 275, 1108-1114.

(9) Dupin, D.; Fujii, S.; Armes, S. P.; Reeve, P.; Baxter, S. M. Langmuir 2006, 22, 3381-3387.

(10) Antonietti, M.; Landfester, K. Prog. Polym. Sci. 2002, 27, 689-757.

(11) Deka, S. R.; Quarta, A.; Di Corato, R.; Falqui, A.; Manna, L.; Cingolani, R.; Pellegrino, T. Langmuir 2010, 26, 10315-10324.

(12) Palioura, D.; Armes, S. P.; Anastasiadis, S. H.; Vamvakaki, M. Langmuir 2007, 23, 5761-5768.

(13) Akamatsu, K.; Shimada, M.; Tsuruoka, T.; Nawafune, H.; Fujii, S.; Nakamura, Y. Langmuir 2010, 26, 1254-1259.

(14) Karg, M.; Lu, Y.; Carbol-Argibay, E.; Pastoriza-Santos, I.; PeÌrez-Juste, J.; Liz-MarzaÌn, L. M.; Hellweg, T. Langmuir 2009, 25, 3163-3167.

(15) Hyeon, T.; Lee, S. S.; Park, J.; Chung, Y.; Bin Na, H. J. Am. Chem. Soc. 2001, 123, 12798-12801.

(16) Fink, J.; Kiely, C. J.; Bethell, D.; Schiffrin, D. J. Chem. Mater. 1998, 10, 922-926.

(17) Cozzoli, P. D.; Pellegrino, T.; Manna, L. Chem. Soc. Rev. 2006, 35, 1195-1208.

(18) Mei, B. C.; Susumu, K.; Medintz, I. L.; Delehanty, J. B.; Mountziaris, T. J.; Mattoussi, H. J. Mater. Chem. 2008, 18, 4949-4958.

(19) Amstad, E.; Zurcher, S.; Mashaghi, A.; Wong, J. Y.; Textor, M.; Reimhult, E. Small 2009, 5, 1334-42.

(20) Amstad, E.; Gillich, T.; Bilecka, I.; Textor, M.; Reimhult, E. Nano Lett 2009, 9, 4042-8.

(21) Sheibat-Othman, N.; Bourgeat-Lami, E. Langmuir 2009, 25, 10121-10133.

(22) Reculusa, S. p.; Mingotaud, C.; Bourgeat-Lami, E.; Duguet, E.; Ravaine, S. Nano Lett. 2004, 4, 1677-1682.

(23) Perro, A.; Nguyen, D.; Ravaine, S.; Bourgeat-Lami, E.; Lambert, O.; Taveau, J.-C.; Duguet, E. J. Mater. Chem. 2009, 19, 4225-4230.

(24) Ruedas-Rama, M. J.; Orte, A.; Hall, E. A. H.; Alvarez-Peza, J. M.; Talavera, E. M. Chemical Communications 2011.

(25) Kirchner, C.; Liedl, T.; Kudera, S.; Pellegrino, T.; Javier, A. M.; Gaub, H. E.; Stolzle, S.; Fertig, N.; Parak, W. J. Nano Lett. 2005, 5, 331-338.

(26) Winter, J. O.; Gomez, N.; Gatzert, S.; Schmidt, C. E.; Korgel, B. A. Colloids Surf., A 2005, 254, 147-157.

(27) Gooding, A. K.; Gomez, D. E.; Mulvaney, P. ACS Nano 2008, 2, 669-676.

(28) Riedinger, A.; Zhang, F.; Dommershausen, F.; Rocker, C.; Brandholt, S.; Nienhaus, G. U.; Koert, U.; Parak, W. J. Small 2010, 6, 2590-2597.
(29) Schmaljohann, D. Adv. Drug Delivery Rev. 2006, 58, 1655-1670.

(30) Gao, W. W.; Chan, J. M.; Farokhzad, O. C. Mol. Pharmaceutics 2010, 7, 1913-1920.

(31) Xu, X.; Friedman, G.; Humfeld, K. D.; Majetich, S. A.; Asher, S. A. Chem. Mater. 2001, 14, 1249-1256.

(32) Zhang, F.; Ali, Z.; Amin, F.; Feltz, A.; Oheim, M.; Parak, W. J. ChemPhysChem 2010, 11, 730-735.

(33) Gazeau, F.; Levy, M.; Wilhelm, C. Nanomedicine 2008, 3, $831-844$.

(34) Figuerola, A.; Di Corato, R.; Manna, L.; Pellegrino, T. Pharmacol. Res. 2010, 62, 126-143. 